\title{
Sobre cambios en la línea de costa entre 1735 y 2011 y la subsidencia en la Bahía de Cartagena de Indias, Colombia
}

\author{
Carlos Alberto Andrade-Amaya ${ }^{1, *}$, Alex José Ferrero-Ronquillo², Hermann León-Rincón², \\ Héctor Mora-Páez ${ }^{3}$, Henry Carvajal-Perico ${ }^{4}$ \\ ${ }^{1}$ Facultad de Oceanografía Física, Escuela Naval de Cadetes “Almirante Padilla”, Cartagena, Colombia \\ ${ }^{2}$ Centro de Investigaciones Oceanográficas e Hidrográficas, CIOH, Cartagena, Colombia \\ ${ }^{3}$ Dirección de Geoamenazas, Servicio Geológico Colombiano, Bogotá, Colombia \\ ${ }^{4}$ Dirección de Geociencias Básicas, Servicio Geológico Colombiano, Bogotá, Colombia
}

Artículo de posesión para el ingreso como miembro de número a la

Academia Colombiana de Ciencias Exactas, Físicas y Naturales el 27 de abril de 2016

\begin{abstract}
Resumen
Se analizó la tendencia del ascenso del nivel del mar en Cartagena con respecto a otros puertos en el mar Caribe en el contexto de los cambios que se aprecian en la línea de costa en la región. La comparación entre mapas del sur de la Bahía de Cartagena elaborados en 1735 y 2011 evidenció la desaparición de islas y zonas de manglar en una amplia zona del área suroeste. La pérdida de zona costera en la Bahía se refleja en una mayor tasa de ascenso del nivel del mar en Cartagena debido a la subsidencia local, lo cual requiere de acciones inmediatas de prevención. Los datos geodésicos de la estación GPS instalada sobre el techo de la edificación del Centro de Investigaciones Oceanográficas e Hidrográficas, $\mathrm{CIOH}$, revelaron una posible subsidencia en la región de la Isla de Manzanillo, la cual podrá contrastarse con los datos que puedan obtenerse de una estación empotrada sobre la superficie terrestre. (C) 2017. Acad. Colomb. Cienc. Ex. Fis. Nat.
\end{abstract}

Palabras clave: Bahía de Cartagena; Subsidencia local; Erosión costera; Geodesia espacial.

On coastline changes between 1735 and 2011 and subsidence in Cartagena Bay, Colombia

\begin{abstract}
We analyzed the trend of sea-level rise in Cartagena with respect to other ports in the Caribbean in the context of coast line changes in the region. The comparison between maps made in 1735 and 2011 of south Cartagena Bay showed the loss of islands and mangrove patches in a large area of the southwest. The loss of coastal zone in the Bay is reflected in a greater trend of sea-level rise in Cartagena due to local subsidence, which requires immediate action. Geodesic data from a GPS station installed on the roof of the Centro de Investigaciones Oceanográficas e Hidrográficas, $\mathrm{CIOH}$, building showed a possible subsidence in the Manzanillo Island region, the can be contrasted as more data will be acquired from the station installed on land. (c) 2017. Acad. Colomb. Cienc. Ex. Fis. Nat.
\end{abstract}

Key words: Cartagena Bay; Local subsidence; Coastal erosion; Space geodesy.

\section{Introducción}

En los ciclos climáticos glaciales e interglaciales el nivel del mar tuvo una variación del orden de 120 m, aproximadamente. Desde entonces, se ha mantenido en un nivel muy cercano al actual, pero con claras tendencias ascendentes como resultado del efecto de fenómenos como el calentamiento del agua de los océanos, el aporte de agua por fusión de glaciares y mantos de hielo y la actividad tectónica en los límites de placa, lo cual genera movimientos verticales (International Panel for Climate Change, IPCC, 2013). En el informe de este organismo se establece una tasa global promedio del cambio ascensional del nivel medio del mar de 1,7 \pm 0,2 $\mathrm{mm}$ por año para todo el siglo $\mathrm{XX}$, de $1,8 \mathrm{~mm}$ por año entre 1961 y 2003, y entre 2,8 y 3,6 mm por año desde 1993, debido, principalmente, al calentamiento global, la fusión de glaciares y el deshielo de Groenlandia y la Antártica.
Las variaciones del nivel del mar son el resultado de diferentes fuentes físicas que usualmente se distinguen por su tiempo de duración, así, para ondas superficiales de gravedad, entre 1 y 20 segundos; para seiches y tsunamis, rangos de minutos a horas; para las mareas, entre medio día y uno, y para los fenómenos geológicos y climatológicos, de uno a diez años y hasta periodos de larga duración. En este contexto, la forma de hacer las diferentes mediciones de tal forma que garanticen la obtención de datos confiables y su correcta interpretación constituye un gran reto (Intergovernmental Oceanographic Commission, IOC/ UNESCO, 2006). Existen varias formas de medición de los

\footnotetext{
*Correspondencia:

Carlos Andrade, candrade@exocol.com

Recibido: 06 de abril de 2016

Aceptado: 21 de febrero de 2017
} 
cambios del nivel del mar, entre las cuales se cuentan los mareógrafos de diferentes tipos de tecnología, las registradas mediante radar altimétrico, así como las mediciones geodésicas hechas mediante sistemas de navegación por satélite (Global Navigation Satellite System, GNSS), y las terrestres de alta precisión. A manera de ejemplo, en la figura 1 se muestra el valor de 2,9 $\pm 0,4 \mathrm{~mm}$ por año resultado de la estimación del cambio global del nivel medio del mar a partir de mediciones de satélites altimétricos de radar hechas entre 1992 y 2016 con la aplicación tecnológica diseñada en el Laboratorio de Altimetría por Satélite de la National Oceanic and Atmospheric Administration de Estados Unidos (NOAA).

Por lo general, las estaciones permanentes de medición del nivel del mar alrededor del mundo se han dedicado principalmente a la medición de las mareas y del nivel medio, lo cual es el principal objetivo del Global Sea Level Observing System (GLOSS), desarrollado bajo el auspicio de la IOC/UNESCO. Para entender adecuadamente los cambios del nivel del mar y las diferencias que puedan presentarse entre diferentes sitios de medición, es necesario tener en cuenta los factores directamente relacionados con la naturaleza del agua, así como los movimientos verticales de la superficie asociados con procesos naturales, tales como los efectos cosísmicos, el ajuste glacial isostático y la tectónica de placas, y los procesos inducidos por el hombre, como la extracción de agua.

\section{Análisis del nivel relativo del mar y de los cambios en la línea costera}

Datos de mareógrafos. Con el mareógrafo instalado en la punta de Castillogrande de la Bahía de Cartagena se recolectaron datos entre el 2 de septiembre de 1951 y el 26 de abril de 1993. Reubicado posteriormente en el muelle del Centro de Investigaciones Oceanográficas e Hidrográficas$\mathrm{CIOH}$, en la Isla de Manzanillo, las observaciones con el mareógrafo se iniciaron el $1^{\circ}$ de mayo de 1993. Estos datos, contenidos en la base de datos de la NOAA, fueron empleados por Torres, et al. (2006) para estimar el aumento del nivel medio del mar en la Bahía de Cartagena, el cual fue de 3,58 mm por año.

Posteriormente, en un nuevo estudio en el cual se empleó el mismo grupo de datos se estableció que en la Bahía de Cartagena se apreciaba una tendencia al aumento relativo del nivel del mar de 4,5 mm por año (Figura 2); sin embargo, dicha información debe considerarse con suma precaución dada la incertidumbre que acarrea el cambio de posición del equipo. En este sentido, en el puerto de Cristóbal en Panamá el registro solamente mostró un aumento de 1,6 mm por año (Andrade, 2008).

Las tendencias del incremento del nivel medio del mar en las costas colombianas también han sido analizadas por Rangel \& Montealegre (2003), Pabón (2003a, 2003b), y Pabón \& Lozano (2005) empleando las series de nivel medio del mar en el Pacífico y el Caribe y los datos de la

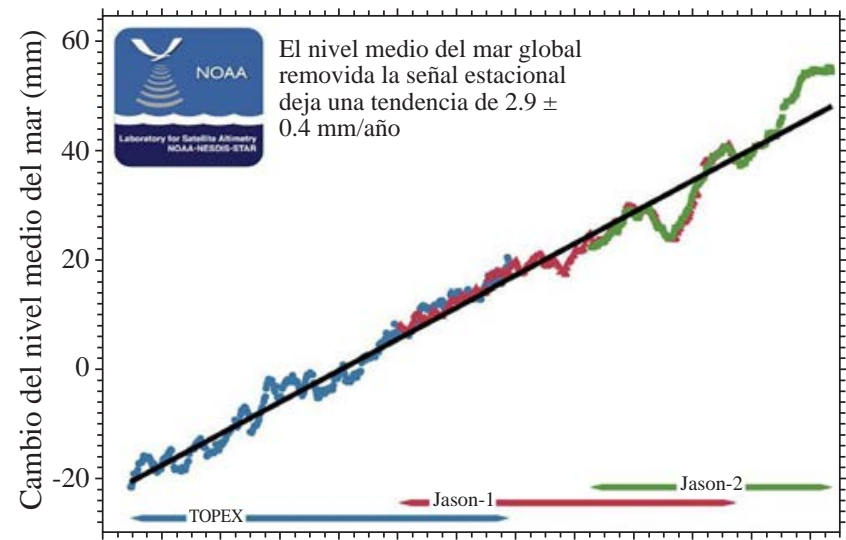

1992199419961998200020022004200620082010201220142016

Figura 1. Cambio global del nivel medio del mar, calculado desde misiones de altimetría satelital para el período 1993-2016. La tendencia lineal muestra que ha subido a razón de 2,9 \pm 0,4 mm/año. Fuente: Laboratorio para Altimetría por Satélite NOAANESDIS-STAR.

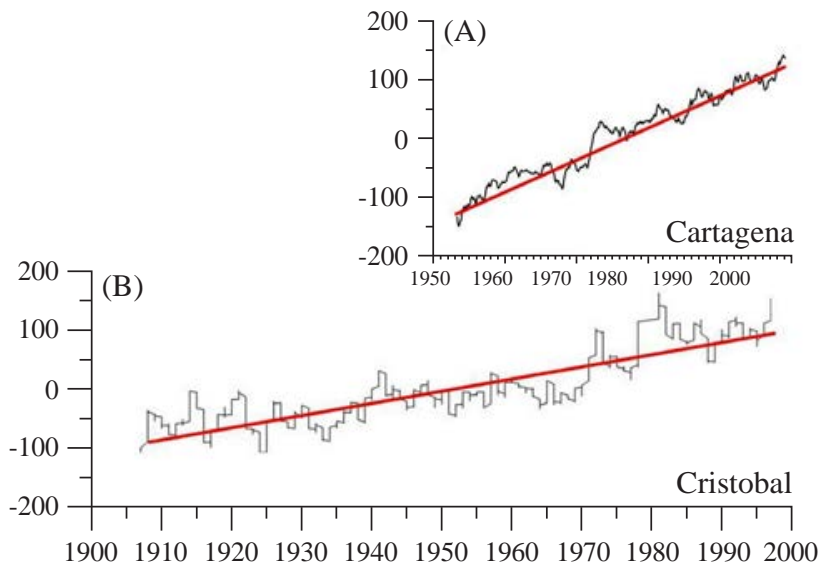

Figura 2. El nivel del mar medido en (A) la Bahía de Cartagena desde 1951 hasta el 2000 y (B) en el puerto de Cristóbal (Panamá) desde 1907 hasta el 2000. Las líneas rojas representan la tendencia lineal de las series. En Cartagena fue de 4,8 mm/año y en Cristóbal fue de 1,6 mm/año, (modificado de Andrade, 2008).

topografía del océano generados por el programa TOPEX/ Poseidon. Dichos trabajos permitieron concluir que entre 1961 y 1990, el incremento regional del nivel del mar tuvo un ritmo de 3 a 5 mm por año en el Pacífico, y de 1 a 2 mm por año en la costa Caribe. Por su parte, el Laboratorio de Altimetría por Satélite de la NOAA, empleando datos obtenidos con radar altimétrico, obtuvo un valor de 2,5 \pm 0,4 mm por año para el período de 1993 a 2016 (Figura 3).

En un análisis reciente de las tendencias para el período de 1908 a 2009, Torres \& Tsimplis (2013) consignaron en Cartagena valores del orden de 5,3 \pm 0,3 mm por año en los últimos 44 años; en Cristóbal, de 1,9 \pm 0,1 mm por año en un periodo de 101,7 años; en Magueyes, Puerto Rico, de 1,3 \pm 0,2 mm por año en 55 años de observación, y en Lime 


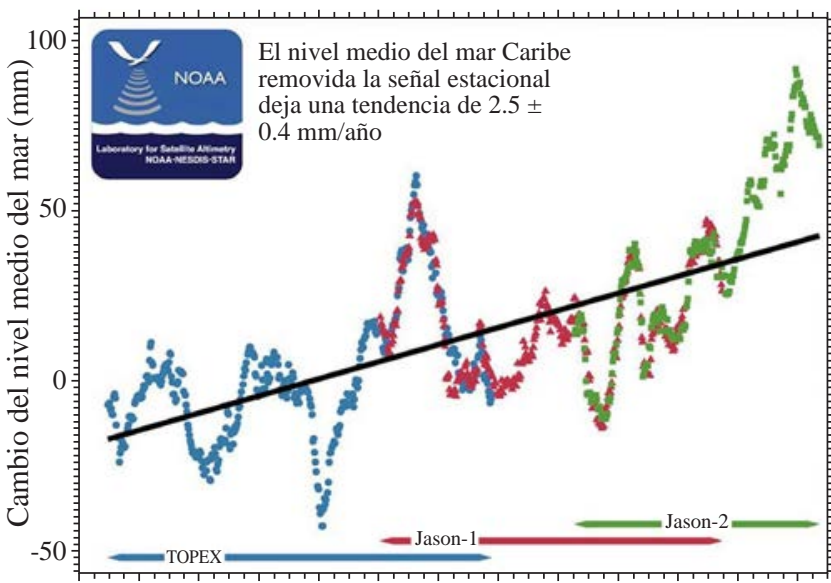

1992199419961998200020022004200620082010201220142016

Figura 3. Estimación del cambio del nivel del mar en el Caribe a partir de datos obtenidos con radar altimétrico para el período 1993-2016 con un valor de 2,5 \pm 0,4 mm/año. Fuente: Laboratorio para Altimetría por Satélite NOAA-NESDIS-STAR

Tree, Cayos de la Florida, de 1,8 \pm 0,5 mm por año en 32,2 años de observación, lo cual les permitió establecer que la tendencia ascensional del nivel relativo en Cartagena es dos o tres veces mayor que en los otros puertos considerados. En un estudio posterior, estos mismos autores (Torres \& Tsimplis, 2014) determinaron la variabilidad temporal de los extremos en los registros de varias estaciones, incluídas las anteriores, con más de 20 años de datos. Para Cartagena solo analizaron el segmento entre 1951 y 1993 debido a la pérdida de continuidad en los datos, así como un segmento más corto para el período de 1994 a 2000 (Figura 4).

Mediciones sobre mapas. Con el propósito de observar los cambios en la línea de la costa y en las profundidades de la Bahía, se utilizaron mapas antiguos que tuvieran el mayor detalle posible para compararlos con la carta náutica del área elaborada por el Centro de Investigaciones Oceanográficas e Hidrográficas (CIOH) en 2011. Los mapas escogidos fueron: a) el mapa levantado por Herrera y Sotomayor, presumiblemente en 1721, en el área de Bocachica, y b) el plano elaborado por Antonio de Ulloa en 1735. El plano de la Bahía de Cartagena fue levantado por Don Juan de Herrera y Sotomayor, ingeniero militar, delineado por Don Carlos de Briones Hoyo y Abarca, y ratificado por Antonio de Ulloa, y corresponde a una escala aproximada de 1:40.200 (Herrera y Sotomayor \& Hoyo y Abarca, 1721) (Figura 5). El segundo mapa, de extraordinario detalle para la época, especialmente porque resalta varias islas de manglar que existían dentro de la Bahía de Cartagena y resuelve las principales formas de los bajos fondos, ofrece varios puntos conspicuos que se pueden utilizar para su georreferenciación. Este mapa manuscrito, coloreado a mano en pluma y tinta, fue elaborado por Herrera y Sotomayor \& Ulloa, 1735), quien se basó en el mapa levantado alrededor de 1721. En él se muestra con gran detalle la Bahía de Cartagena de Indias y el área costera adyacente a la actual ciudad de Cartagena, Colombia. El mapa está orientado por una rosa de los vientos con el Norte apuntando hacia la izquierda y la longitud se estableció con relación al Real Observatorio Astronómico de Tenerife, en las islas Canarias. Los sondeos y las líneas punteadas indican la batimetría. Como se aprecia en el mapa, el sector de la Bahía de Cartagena bajo observación se encuentra al sur de la isla de Tierrabomba y al norte de la isla de Barú, y se caracteriza por tener una línea de costa defendida por manglares casi en su totalidad. En la figura 6 se presenta este mapa, el cual fue debidamente georreferenciado para los propósitos de este trabajo. Como referencia moderna se utilizó la carta náutica COL 264 de la Bahía de Cartagena, en su tercera edición (2011), la cual muestra la línea de costa más actualizada y sirvió para observar los cambios ocurridos en los casi tres siglos que median entre esta y los mapas antiguos.

Dichos mapas se digitalizaron empleando 20 puntos de control distribuidos en toda el área para la georreferenciación mediante el programa ArcGis 10.0. Las líneas de la costa se compararon entre sí y los colchones de manglar en el plano de Herrera y Sotomayor y los dibujados por Herrera y Sotomayor \& Ulloa (1735) se superpusieron sobre la carta náutica No. 264 elaborada por el CIOH de la Dirección General Marítima (Figura 7).

Mediciones geodésicas espaciales por GPS. Estación Cartagena. La estación Cartagena, denominada CART, forma parte de la red MAGNA-ECO del Instituto Geográfico “Agustín Codazzi” de Colombia. Es una estación geodésica GPS instalada el 2 de marzo de 2000 en el techo de una de las edificaciones que conforman el $\mathrm{CIOH}$ en la Isla de Manzanillo en Cartagena. Los datos de esta estación, almacenados en SONEL (http://www.sonel.org), centro de datos del sistema global de navegación por satélite (global navigation satellite system, GNSS) del GLOSS, fueron empleados por Santamaría-Gómez (2010) para obtener un valor de velocidad vertical de $-1,43 \pm 0,35 \mathrm{~mm}$ por año, correspondiente a la solución reprocesada conocida como URL4, del Consorcio Centro de Análisis TIGA de la Universidad de La Rochelle, Francia, a partir de más de 300 estaciones GPS, 200 de las cuales cuentan, además, con mareógrafos. Las velocidades se estimaron a partir de series de tiempo mensuales de posición en un período de 13 años (entre 1996 y 2009), y se expresaron en el International Terrestrial Reference Frame, ITRF2005. Posteriormente, Santamaría-Gómez, et al. (2012) las reprocesaron y obtuvieron el campo de velocidades de 326 estaciones distribuídas globalmente, 200 de ellas con mareógrafos, bajo la solución denominada URL5, la cual fue el resultado de un nuevo análisis de 16 años de datos de GPS (entre 1995 y 2010), y se expresó en el ITRF2008. Las velocidades GPS se obtuvieron como paso final del análisis de las soluciones globales de red, empleando el programa GAMIT-GLOBK (Herring, et al., 2010a, 2010b), con una estrategia de procesamiento diferencial de GPS y una solución a largo plazo mediante el programa CATREF, el cual permite la 
(A) Percentiles del nivel del mar en Magueyes

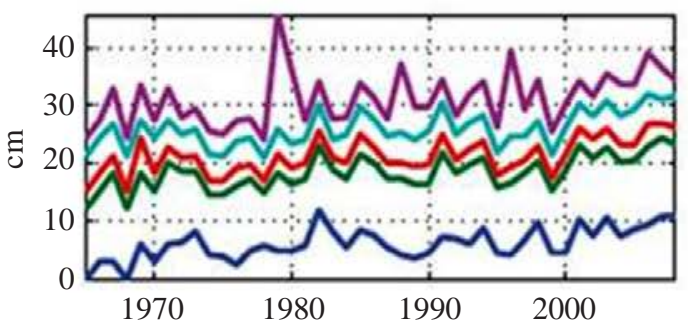

Percentiles del nivel del mar en Lime Tree

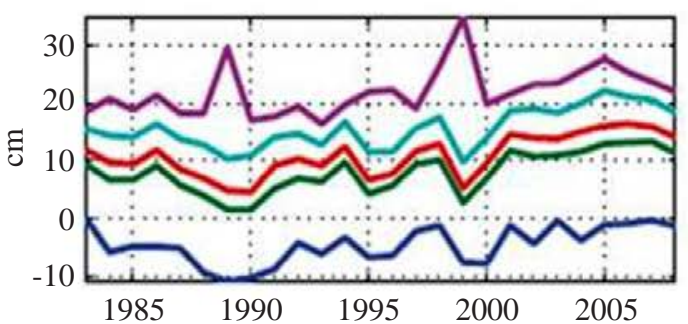

Percentiles del nivel del mar en Pto. Cortés

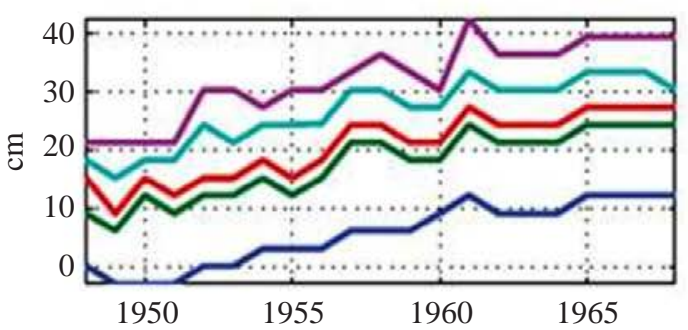

Percentiles del nivel del mar en Cartagena

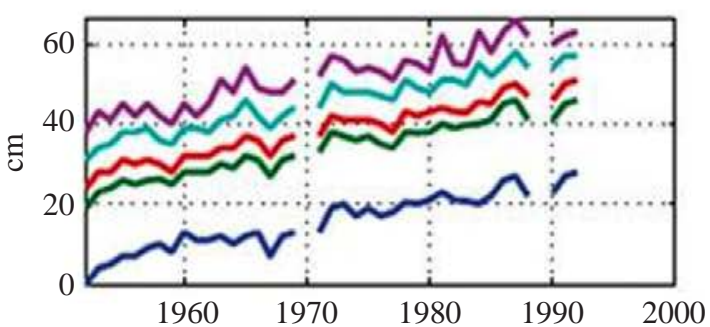

Percentiles del nivel del mar en Cristobal

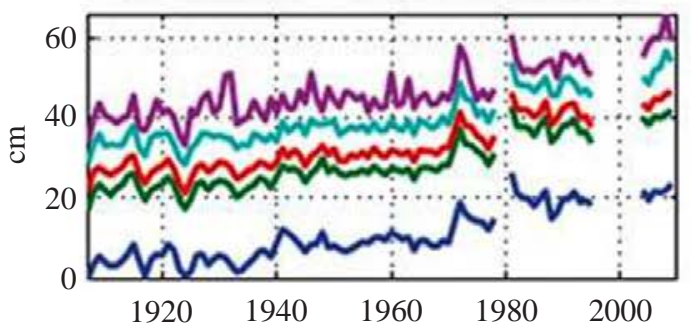

(B) Percentiles del residuo sin marea en Magueyes

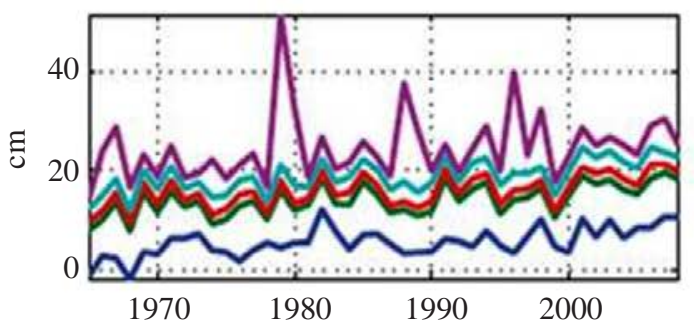

Percentiles del residuo sin marea en Lime Tree

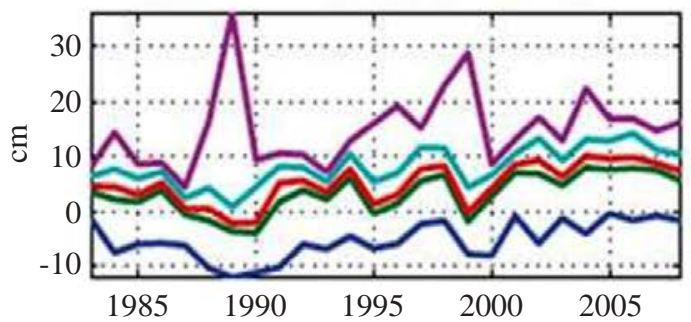

Percentiles del residuo sin marea en Pto. Cortés

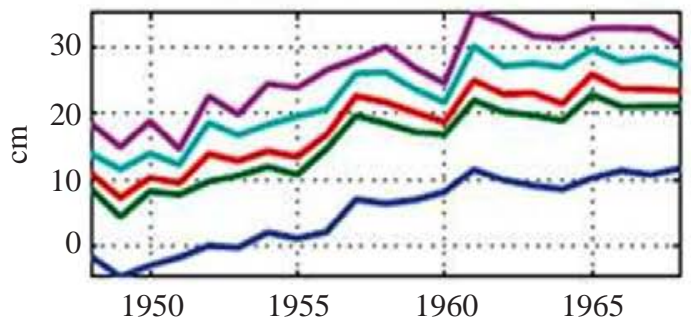

Percentiles del residuo sin marea en Cartagena

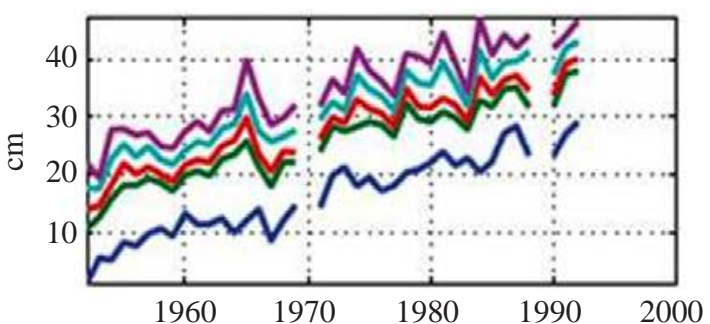

Percentiles del residuo sin marea en Cristobal

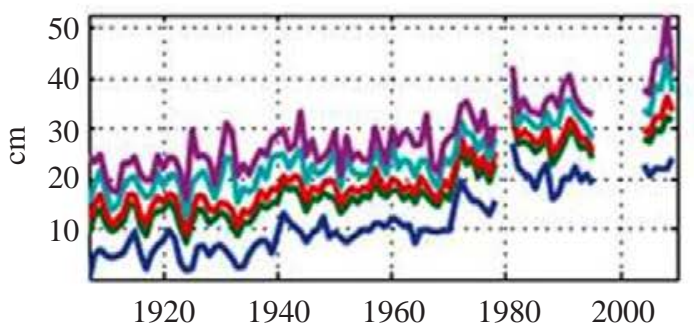

Figura 4. Percentiles 50, 90, 95 y 99,9, de la parte inferior a la superior en cada gráfica, calculados con (A) observaciones y (B) residuos no mareales en Magueyes, Lime Tree, P. Cortes, Cartagena y Cristóbal. Las series de tiempo son referenciadas al primer año de nivel medio de mar. Tomada de Torres \& Tsimplis (2014)

combinación y el análisis de marcos de referencia terrestres. Al reprocesar los datos, Santamaría (2012) obtuvo una velocidad vertical de $-2,19 \pm 0,54 \mathrm{~mm}$ por año con base en datos de 8,54 años recolectados en la estación CART.
Estación CIOH. Bajo el marco del proyecto de investigación e innovación tecnológica conocido como GeoRED (Red Nacional de Estaciones del GNSS con propósitos geodinámicos), el Grupo de Investigaciones Geodésicas 


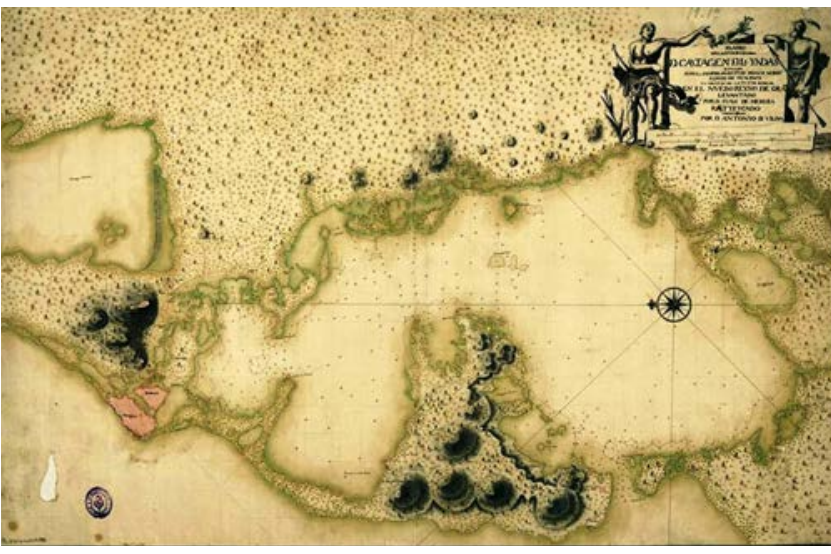

Figura 5. Plano de la Bahía de Cartagena, levantado por Don Juan de Herrera y Sotomayor, delineado por Don Carlos de Briones Hoyo y Abarca, y ratificado por Antonio de Ulloa; corresponde a una escala aproximada de 1:40.200, (Herrera y Sotomayor, 1721). Fuente: Biblioteca Nacional de España

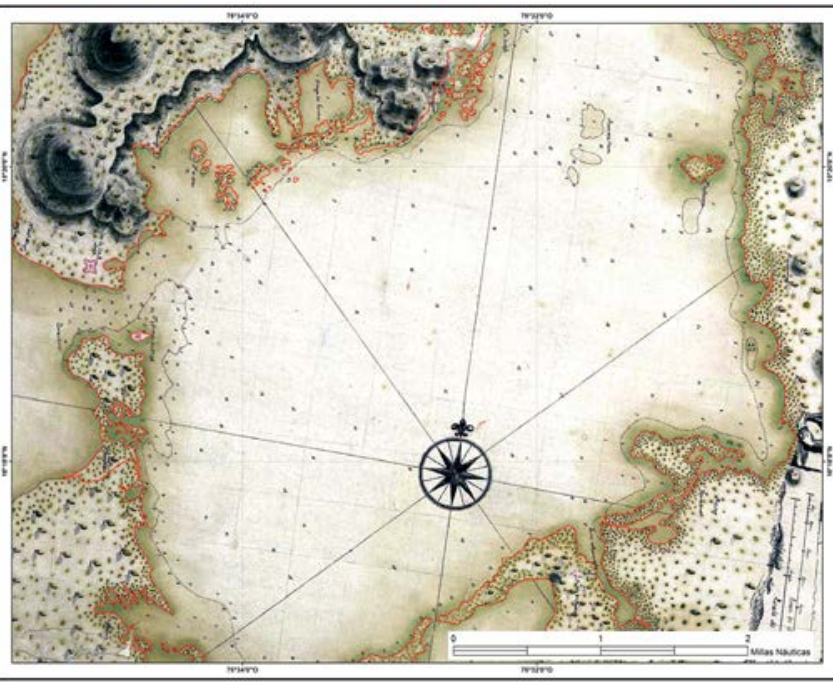

Figura 6. Recuadro del área suroeste de la Bahía de Cartagena en el plano de Herrera y Sotomayor \& Ulloa de 1735 debidamente georreferenciado.

Espaciales del Servicio Geológico Colombiano, GIGESGC, instaló una estación geodésica espacial del GNSS en predios del $\mathrm{CIOH}$ a finales de octubre de 2014. La instalación se hizo directamente sobre el terreno, con el fin de garantizar estabilidad en la posición de la antena y obtener observaciones de gran confiabilidad. La base de la antena está soportada por una estructura metálica en acero inoxidable cuyo mástil central, sobre el cual se coloca directamente la antena del GNSS, fue empotrado a una profundidad de 19,4 m, y sobresale 1,4 m de la superficie. El procesamiento diario de los datos se hace con el programa GIPSY-OASS II (Zumberge, et al., 2007), desarrollado por el Jet Propulsion Laboratory de la NASA (JPL-NASA), cuyos resultados son procesados, a su vez, con el programa HECTOR (Bos, et al., 2013), mediante el cual se generan las series de tiempo geodésicas y se estiman las velocidades.

\section{Resultados y análisis}

Información de los mareógrafos. El análisis de los resultados de los mareógrafos sugiere que la elevación del mar en el área de la Bahía de Cartagena se está incrementando en una proporción cercana al doble con respecto a los datos obtenidos en los costados del mar Caribe. Sería de esperar que la superficie libre del mar tuviera medidas parecidas en sitios relativamente cercanos, pero el hecho de que las tendencias del nivel del mar medidas en los puertos de Cartagena y Cristóbal, separados solamente por 300 millas, sean tan diferentes indicaría que en el sector de la Bahía de Cartagena existe un elemento adicional causado por el cambio climático que incide en un mayor y más veloz aumento del nivel del mar. Para tratar de entender las posibles causas de esta diferencia, se procedió a evaluar la geomorfología de la Bahía de Cartagena mediante el análisis de las variaciones en la zona costera a partir de la comparación de las líneas de costa y de las profundidades medidas en diferentes épocas, y, asimismo, se inició el análisis de las mediciones geodésicas espaciales del GNSS disponibles en el sector.

Línea de costa. Por otra parte, el análisis de la línea de costa demarcada en la carta de 1735 en el sur de la Bahía de Cartagena, comparada con la carta de navegación actual, demostró que la zona costera ha perdido 342,4 hectáreas de terreno, especialmente de los llamados "colchones de mangle", zonas de manglar que conformaban el borde norte de la isla de Barú dentro de la Bahía, como puede observarse en el mapa de la figura 8. El mapa de la figura 5 permite apreciar que la zona del sur estaba cubierta por una franja amplia de manglar cuya punta occidental se extendía hacia el norte sobre la boca de Ciénaga Honda. En la esquina suroeste de la Bahía existió una laguna protegida por islas y colchones de manglar que hoy son bajos fondos de $1,5 \mathrm{~m}$ de profundidad, en tanto que en el sector de Bocachica lo más significativo es la desaparición casi total de Isla Abanico.

Isla Abanico era una isla de 42 hectáreas en 1735 (Figura 9a), muy importante para la defensa de la Bahía; en abril de 1741 allí se instalaron baterías de artillería, muy efectivas para la protección combinada de los fuertes (Blas de Lezo, 1741). Esta isla apareció en los mapas con su misma forma hasta finales del siglo XIX; empezó a desaparecer en los mapas del siglo pasado y hoy es apenas una franja de manglar de 3,09 hectáreas (Figura 9b). Andrade \& Franco (2016) han descrito los problemas de dicha isla y de los manglares entre Bocachica y Caño de Loro.

En resumen, la comparación de estas dos cartas náuticas evidenció cómo la línea de costa del sur de la Bahía de Cartagena ha cambiado: varias hectáreas de terreno de manglar y de islas han desaparecido en todo su perímetro, especialmente en el punto más abrigado contra el oleaje y las condiciones extremas del mar, lo cual indica un efecto 


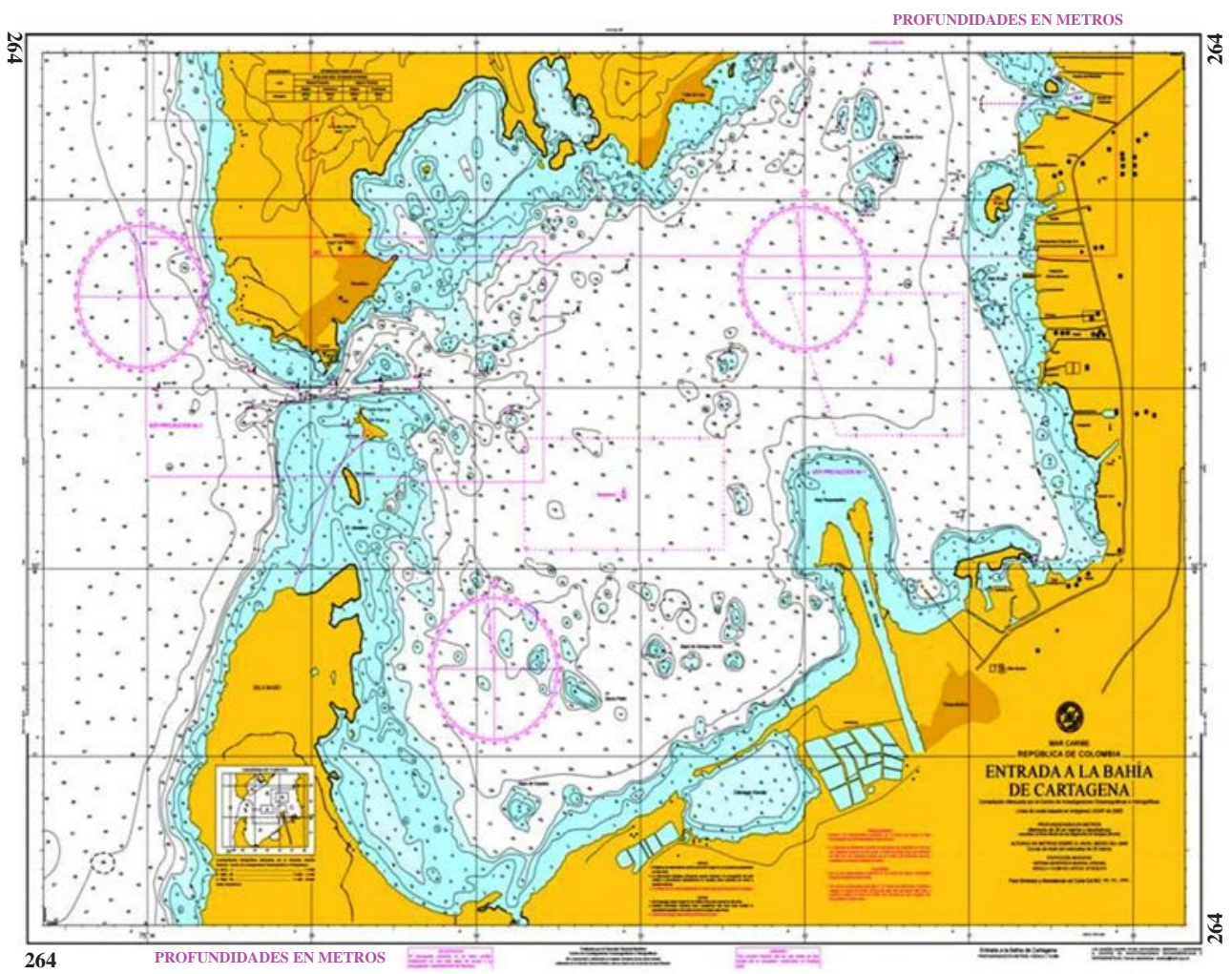

Figura 7. Carta Náutica 264, 3a edición, de 2011 cuya línea de costa fue utilizada como la más reciente para la comparación. Fuente: DIMAR, CIOH, 2011

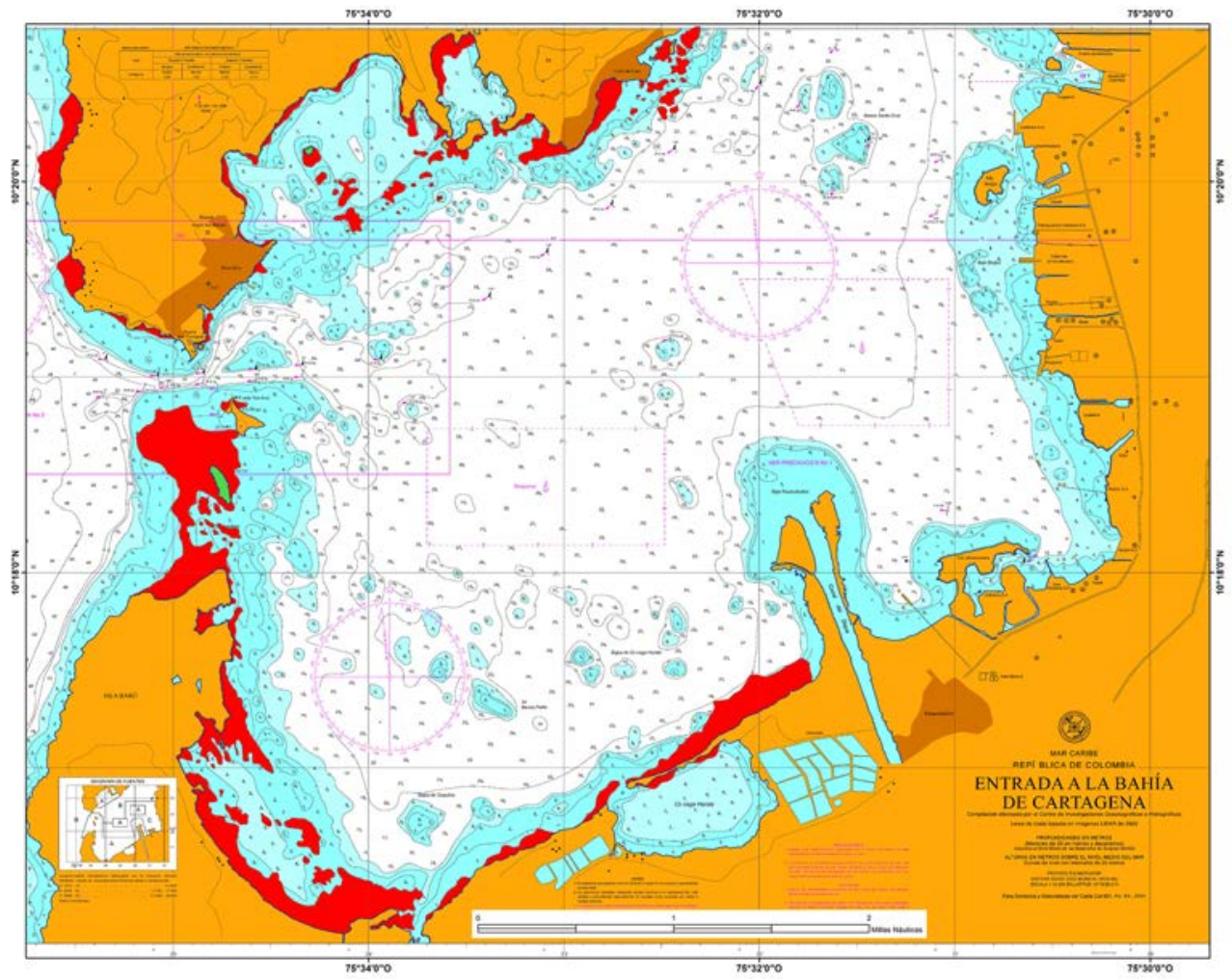

Figura 8. Tomando como referencia la Carta Náutica 264, se señalan los bloques en rojas que corresponden a zonas de área costera emergidas y visualizadas en los mapas antiguos, que no existen hoy. 
A.

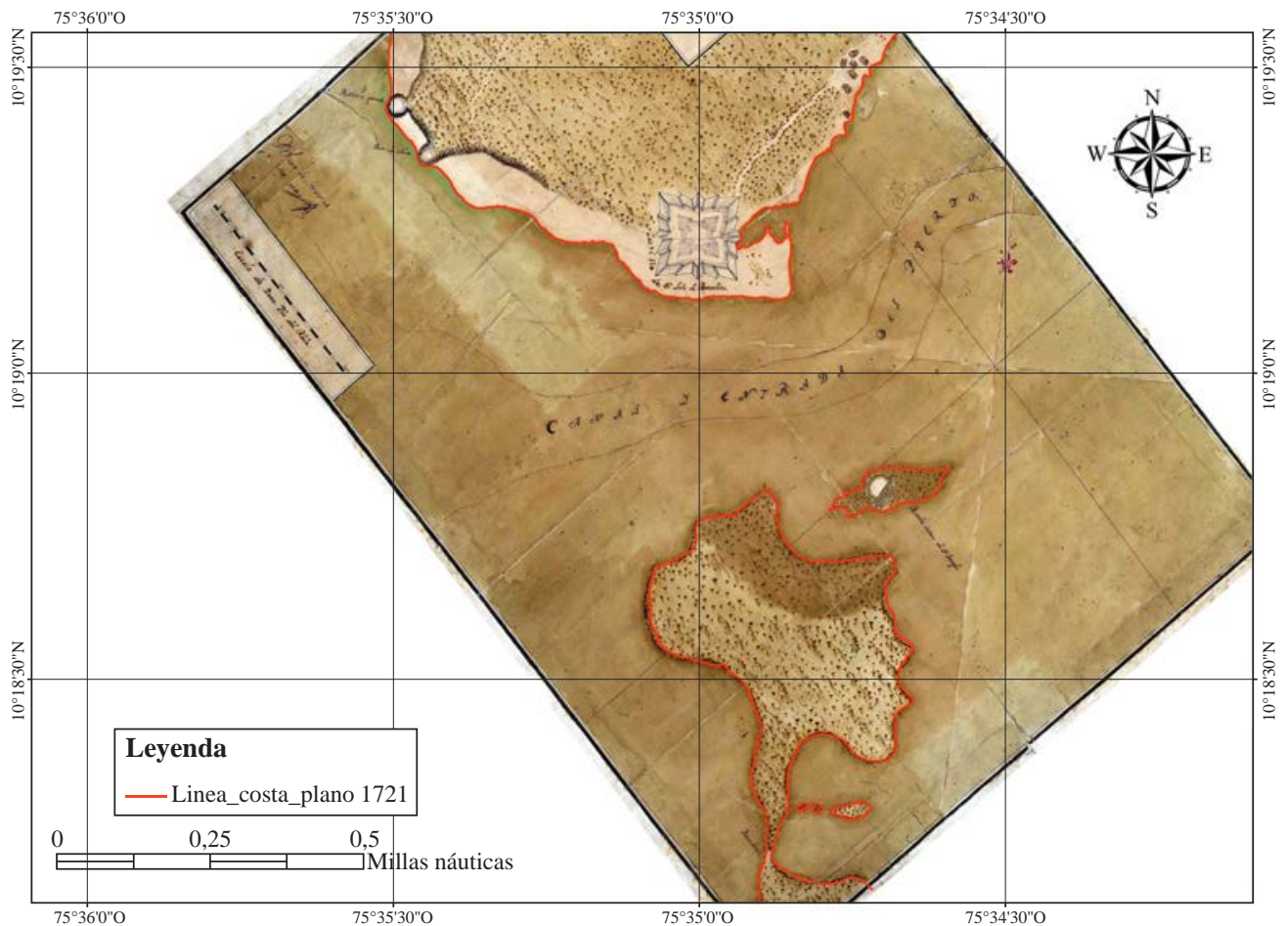

B.

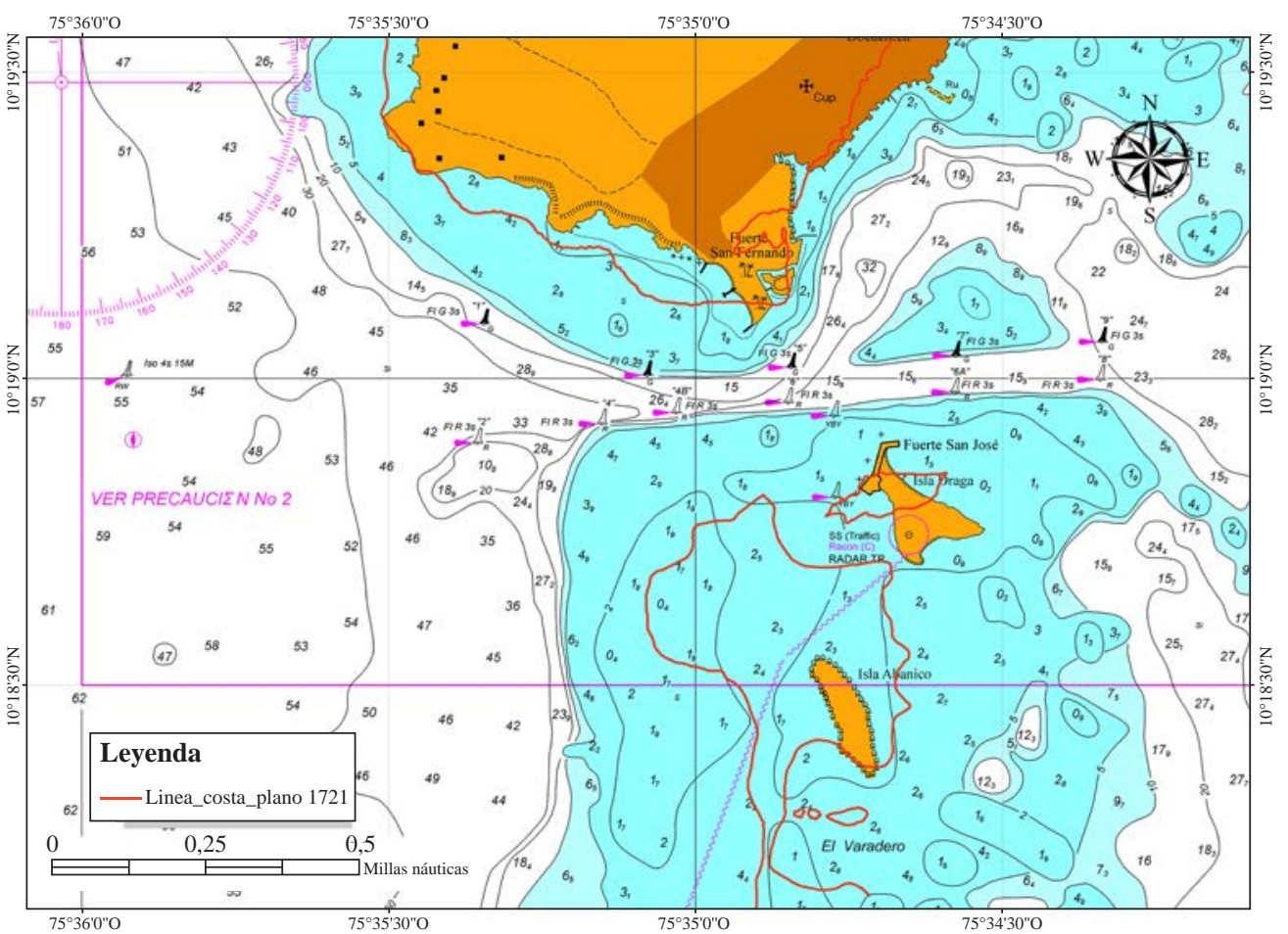

Figura 9. El mapa 9a, (izq), de Herrera y Sotomayor de 1721 muestra Isla Abanico al sur de Bocachica. El mapa 9b (der) señala la línea de costa georreferenciada de 9a superpuesta a la carta batimétrica del CIOH (2011); los espacios entre la línea roja y la costa actual son los terrenos y manglares que existieron en 1735 y que ya han desaparecido bajo el mar.

local diferente al de los procesos costeros exteriores a la Bahía, con dinámicas similares a la erosión costera del frente de playa influenciado por el oleaje normal y los fenómenos extremos.
Información geodésica. En el SONEL se dispone de la solución ULR6 de los datos de la estación CART, solución que corresponde al procesamiento de 749 estaciones del GNSS a nivel global con datos del periodo entre 1995 y 2014, 
y recurre a los modelos y correcciones más actualizadas (Gravelle, et al., 2015), es decir, datos más depurados que los de las soluciones anteriores. Esta solución fue empleada por el GIGE-SGC bajo el marco de GeoRED para elaborar la respectiva serie de tiempo con el programa HECTOR ya mencionado. Mediante el procesamiento de 1.841 datos de dicha solución, correspondientes al periodo entre el 3 de marzo de 2000 y el 29 de diciembre de 2013, se obtuvo la tendencia vertical de la velocidad de $-1,78 \pm 0,43 \mathrm{~mm}$ por año (Figura 10), valor intermedio comparado con los resultados obtenidos por Santamaría-Gómez en el 2010 y por Santamaría-Gómez, et al. en el 2012. Lamentablemente, se estableció que en esta estación ha habido una gran pérdida de datos en diversos períodos de tiempo; así, el número de datos esperados para el tiempo de observación (marzo 3 de 2000 a diciembre 29 de 2013), era de 5.079, pero solo estaban disponibles 1.841 datos. Por tal motivo, en el procesamiento de la serie de tiempo se empleó la estimación por máxima verosimilitud calculada mediante el método de covarianza total, pues más de $60 \%$ de los datos de la muestra total no estaban disponibles. Se tuvieron en cuenta los parámetros de señal estacional, desfase, factor intercuartil de 3,0, en tanto que la fecha de referencia fue el 29 de junio de 2004; además, se consideró un salto (offset), el cual se marcó en la gráfica de la serie de tiempo, con lo cual se eliminaron ocho datos atípicos. Con propósitos de comparación, solamente se estimó la tendencia para el período entre 2000 y 2008, con el fin de verificar el impacto de la carencia de datos entre el 2008 y el 2013, obteniéndose una tendencia vertical de -1,88 \pm 0,44 mm por año, concordante con el valor obtenido para el período entre 2000 y 2013 (Figura 11).

Por otra parte, los 826 datos obtenidos del total de 836 esperados entre el 29 de octubre de 2014 y el 11 de febrero de 2017 (2,3 años) en la estación CIOH fueron procesados por el GIGE del SGC con los programas GIPSY-OASIS II y HECTOR. En este estudio se empleó el método AmmarGrag, ya que el número de datos faltantes no era considerable, $\mathrm{y}$ se obtuvo un valor de $0,06 \pm 1,54 \mathrm{~mm}$ por año (Figura 12). Además, se consideró un salto por actualización del programa firmware en el receptor geodésico.

HECTOR es un programa especializado para el estudio de series de tiempo del GNSS que permite estimar la línea de tendencia en series de tiempo con correlaciones temporales de ruido. Es un programa dinámico que solo acepta ruido estacionario con propiedades de ruido constantes y permite el uso rápido de operaciones matriciales, con lo cual se obtiene

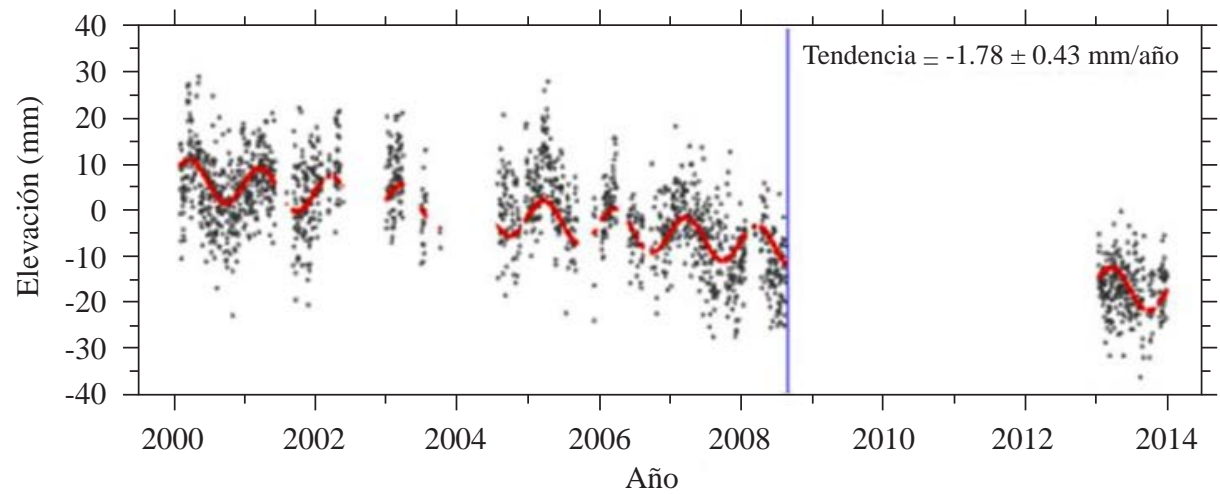

Figura 10. Serie geodésica de tiempo de la estación CART generada a partir de la solución disponible en SONEL empleando el software HECTOR para el período 2000-2013

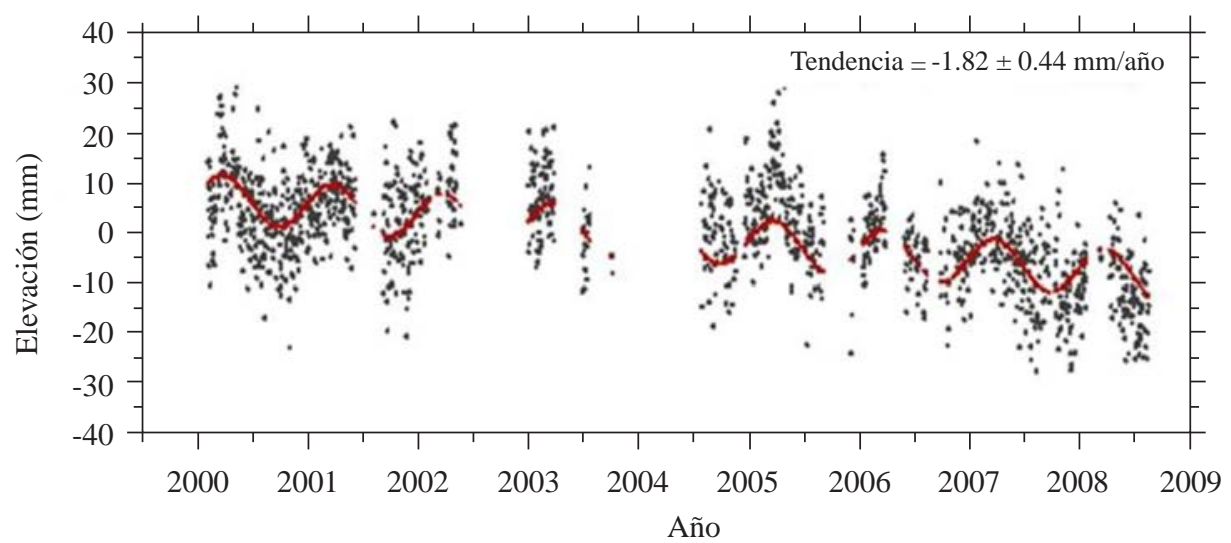

Figura 11. Serie geodésica de tiempo de la estación CART generada a partir de la solución disponible en SONEL empleando el software HECTOR para el período 2000-2008 


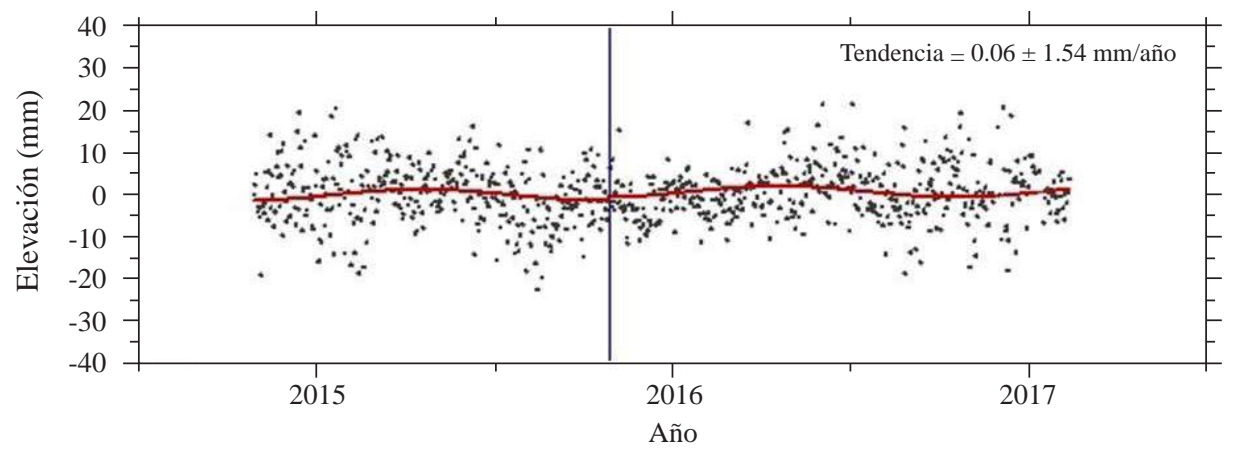

Figura 12. Serie geodésica de tiempo de la estación $\mathrm{CIOH}$ generada a partir de los datos disponibles empleando el software HECTOR.

una reducción en el tiempo de procesamiento en comparación con otros programas similares. Para la generación de series de tiempo se aplican los modelos de ruido Power-Law y White Noise y la estimación numérica de máxima verosimilitud mediante los métodos AmmarGrag o Full Covariance para el cálculo, dependiendo del caso. Para valorar la calidad del modelo de ruido elegido se aplican los criterios de información de Akaike (Akaike Information Criteria, AIC) y los bayesianos (Baysian Information Criteria, BIC). En una serie de tiempo se observa el comportamiento de la estación durante el tiempo de observación y se estima un valor de tendencia, así como la dirección de desplazamiento en cada uno de sus tres componentes. Los datos atípicos se eliminan usando un factor intercuartil, y la tendencia se estima mediante un modelo lineal y parámetros de ruido establecidos a partir de la combinación de los modelos mencionados. Se consideran además otros tipos de eventos, tales como los cambios en los equipos geodésicos, actualización del firmware, reorientación de la antena, y fenómenos naturales como los sismos, los cuales pueden tener alguna injerencia en la generación de las series de tiempo, pues afectan las posiciones diarias de las estaciones del GNSS permanentes; asimismo, se estima el valor del salto en caso de que este altere el comportamiento de la serie de tiempo.

\section{Discusión}

Los análisis efectuados permitieron establecer una clara tendencia ascensional del nivel medio del mar, con un valor entre 2,8 y 3,6 mm por año desde 1993, calculado a partir de los datos de los mareógrafos. Los datos de altimetría satelital registrados entre 1993 y 2016 confirman este valor, con una estimación del cambio global de 2,9 mm por año; en el caso particular de la región Caribe, los datos del radar altimétrico arrojaron un valor de $2,5 \pm 0,4 \mathrm{~mm}$ por año para el mismo período.

Por otra parte, son varios los autores que han hecho estimaciones a partir de los datos de los mareógrafos, así como comparaciones con las estaciones cercanas, para determinar las variaciones del nivel del mar en la Bahía de Cartagena, lo cual permite concluir que los valores obtenidos en esta zona son dos a tres veces superiores a los obtenidos en otras estaciones.
A partir de los datos de la estación geodésica GPS CART, Santamaría (2010) y Santamaría, et al. (2012) estimaron valores de subsidencia en el rango de $-1,43 \pm$ 0,35 a $-2,19 \pm 0,54 \mathrm{~mm}$ por año, correspondientes a las soluciones ULR4 y ULR5, respectivamente. En este trabajo se hizo el cálculo empleando los datos de la solución ULR6, los cuales arrojaron valores de $-1,78 \pm 0,4$ y $-1,88 \pm$ 0,44 mm por año para dos períodos de tiempo diferentes. Aunque localizada sobre el techo de un edificio del $\mathrm{CIOH}$, esta estación ha permitido registrar datos que apuntan a la existencia de subsidencia en un valor cercano a los 2 mm por año. Por su parte, los datos de la estación GPS, conocida como CIOH e instalada por el Servicio Geológico Colombiano, no presentaron una tendencia tan clara como la anotada, pues fue del orden de $0,06 \pm 1,54 \mathrm{~mm}$ por año; se observó que el margen de incertidumbre es alto con respecto al valor obtenido. En este sentido es importante considerar que las series geodésicas de tiempo que emplean datos de posición GPS contienen señales tectónicas y no tectónicas, así como otros errores no modelados que afectan la estimación de las velocidades de las estaciones y sus incertidumbres, por lo cual es necesario eliminar o reducir dichas influencias. Además, en muchos estudios teóricos y usos operacionales las señales estacionales deben separarse de otras señales asociadas a movimientos de periodos largos y seculares (He, et al., 2017). Por lo tanto, la comprensión de los efectos estacionarios es complicada, ya que existe la posibilidad de que diferentes fuentes de ruido se superpongan. En este orden de ideas, el análisis de los efectos estacionarios requiere de tiempos prolongados de observación, con el fin de extraer los parámetros asociados a la deformación de la corteza terrestre. En un estudio de la influencia de las señales anuales en la incertidumbre de las velocidades derivadas del GPS se señala que los sesgos en la velocidad son despreciables después de 4,5 años de observación, y que las velocidades obtenidas a partir de series de tiempo menores a 2,5 años tienen un considerable sesgo de tendencia (Blewitt \& Lavallée, 2002). Con base en el incremento de las observaciones de las series de tiempo, Santamaría-Gómez, et al., (2011), por su parte, establecieron que los efectos de las señales periódicas en la estimación de las velocidades era pequeña, menor de $0,2 \mathrm{~mm}$ por año. 
Por consiguiente, se necesita un tiempo de observación más prolongado en la estación $\mathrm{CIOH}$, en especial por su condición de estar empotrada en el terreno.

Sin embargo, si se colocan en contexto las medidas de subsidencia calculadas a partir de los datos geodésicos del GPS y las mediciones que han estimado el ascenso del nivel del mar en por lo menos 2,8 mm por año (IPCC, 2013), se podría considerar su efecto conjunto en la determinación del nivel relativo del mar en la Bahía de Cartagena, el cual es mayor al obtenido en los puertos vecinos, estimados por Torres \& Tsimplis (2013) en el orden de 5,3 \pm 0,3 mm por año en los últimos 44 años. El hecho de que los mayores cambios en la línea de costa se hayan encontrado en el área interior de la Bahía, donde el proceso costero es menos intenso en comparación con la costa externa, obliga a examinar otros factores que puedan explicar estos cambios. Tales factores podrían ser efectos geológicos locales, tales como las emanaciones de gas o el diapirismo de lodo, que podrían estar activos en el área sur de la Bahía de Cartagena.

Por otra parte, se ha sugerido que el mayor incremento del nivel del mar en Cartagena también puede deberse a la compactación de la zona por la intensa urbanización en Castillogrande durante la segunda mitad del siglo pasado (Nicolae, et al., 2008), lo cual exigiría desarrollar trabajos para resolver si los movimientos de la corteza se restringen a Castillogrande o si se extienden a otras zonas.

Es evidente que los terrenos desaparecidos, detectados mediante la comparación cartográfica efectuada, corresponden muy bien a bajos fondos que hoy se encuentran a un metro de profundidad aproximadamente, tanto en los extensos colchones de manglares como en la línea de costa del sur de la Bahía de Cartagena.

La Bahía de Cartagena de Indias se considera una laguna costera de origen tectónico cuya barrera corresponde a la isla de Tierrabomba, la cual separa las aguas de la laguna de las de mar abierto (Gayet \& Vernette, 1989). Su conformación, con dos zonas de intercambio de aguas en Bocagrande y Bocachica, se puede atribuir a la tectónica vertical asociada con la común presencia de diapirismo de lodo en la región centro y suroccidental del Caribe colombiano.

Este proceso de deformación se debe a la presencia de materiales de características plásticas con alto contenido de gases en la profundidad, los cuales migran lateralmente debido tanto al contraste de densidad entre ellos y la cobertura superior mucho más densa como a la dinámica tectónica relacionada con la convergencia de las placas Nazca, Suramérica y Caribe (Duque, 1979; Duque-Caro, 1984; Kellogg, et al., 2005; Mantilla, et al., 2009). Se considera que el diapirismo de lodo es responsable de la conformación geomorfológica actual de la zona costera entre Barranquilla y el golfo de Urabá, así como de la presencia de "volcanes de lodo” en la región continental y submarina cercana (Briceño \& Vernette, 1992; Carvajal, 2016).

Las geoformas presentes en los alrededores de la Bahía de Cartagena, como las plataformas de arrecifes localmente basculadas del cerro de La Popa y Turbaco, los dos niveles de terrazas marinas en Tierrabomba y el semi-paleoatolón asociado con el Cerro Albornoz (Carvajal, 2016), son manifestaciones de esta dinámica de levantamientos y subsidencia diferencial que se inició en el Plioceno, hace aproximadamente cuatro millones de años, y aún prevalece en la región (Figura 13). De hecho, el análisis paleontológico del registro fósil del nivel más bajo de terrazas marinas en Tierrabomba permitieron a Martínez, et al., (2010) establecer una tasa de levantamiento del orden de 1,7 mm por año en el sector nororiental de la isla.

Se considera que las zonas bajas asociadas localmente con sinclinales basculados y hundidos en el norte de la isla de Barú y las llanuras costeras intermareales en el costado oriental de la Bahía de Cartagena, se originaron por el mismo proceso de diapirismo de lodo. Igualmente, los bajos de arrecifes y cuerpos coralinos costeros vivos, localmente colonizados de manglar en las riberas de la Bahía de Cartagena de Indias, se asocian al mismo proceso.

Los “volcanes de lodo" son otra de las manifestaciones en superficie del fenómeno de diapirismo de lodo en los alrededores de la Bahía de Cartagena. Estos cerros de 1 km de diámetro y 40 a 100 m de altura, con bocas de diferentes tamaños y formas en la parte superior, se formaron por deformación y por la acumulación de lodos expulsados durante procesos eruptivos. En la región cercana a la Bahía de Cartagena se encuentran los “volcanes de lodo” de Santa Ana Barú, Membrillal, y el Rodeo, este último con registros de erupción a finales del 2013 (Carvajal \& Mendivelso, 2017; Carvajal \& Calderón, 2013).

Estos procesos de expulsión violenta de lodo y gas metano son manifestación del estado de compresión al cual están sometidos los materiales diapíricos en profundidad. Según Carvajal (2017a), los procesos eruptivos se presentan con una recurrencia de 15 a 20 años, lo cual concuerda con las manifestaciones en zonas cercanas al norte de Cartagena.

Con base en el análisis de la información gravimétrica y aero-magnetométrica, Obando (2010), citado en Carvajal (2017b), detectó anomalías atribuibles a la aparición de un cuerpo diapírico en profundidad en la región de Mamonal, en el sector oriental de la Bahía de Cartagena. Recientemente se han registrado emanaciones de lodo y gases (pockmarks) en la bahía misma, lo cual es otro indicador de la dinámica del diapirismo en la región y de su influencia en la estabilidad de las líneas de costa.

El origen del complejo de arrecifes de islas del Rosario está asociado con el diapirismo de lodo (Vernette, 1985; Vernette, et al., 1990; Vernette, et al., 1992). Debe señalarse, también, que la presencia de emanaciones de fluidos y gases en zonas marinas constituye una fuente de nutrientes y explica la aparición y proliferación de comunidades bénticas con un desarrollo anormal de sus individuos, lo cual se ha comprobado en varios sitios de la plataforma continental aledaños a Cartagena (Vernette, et al., 1988). Esta situación se evidencia en los bajos de Salmedina, al 


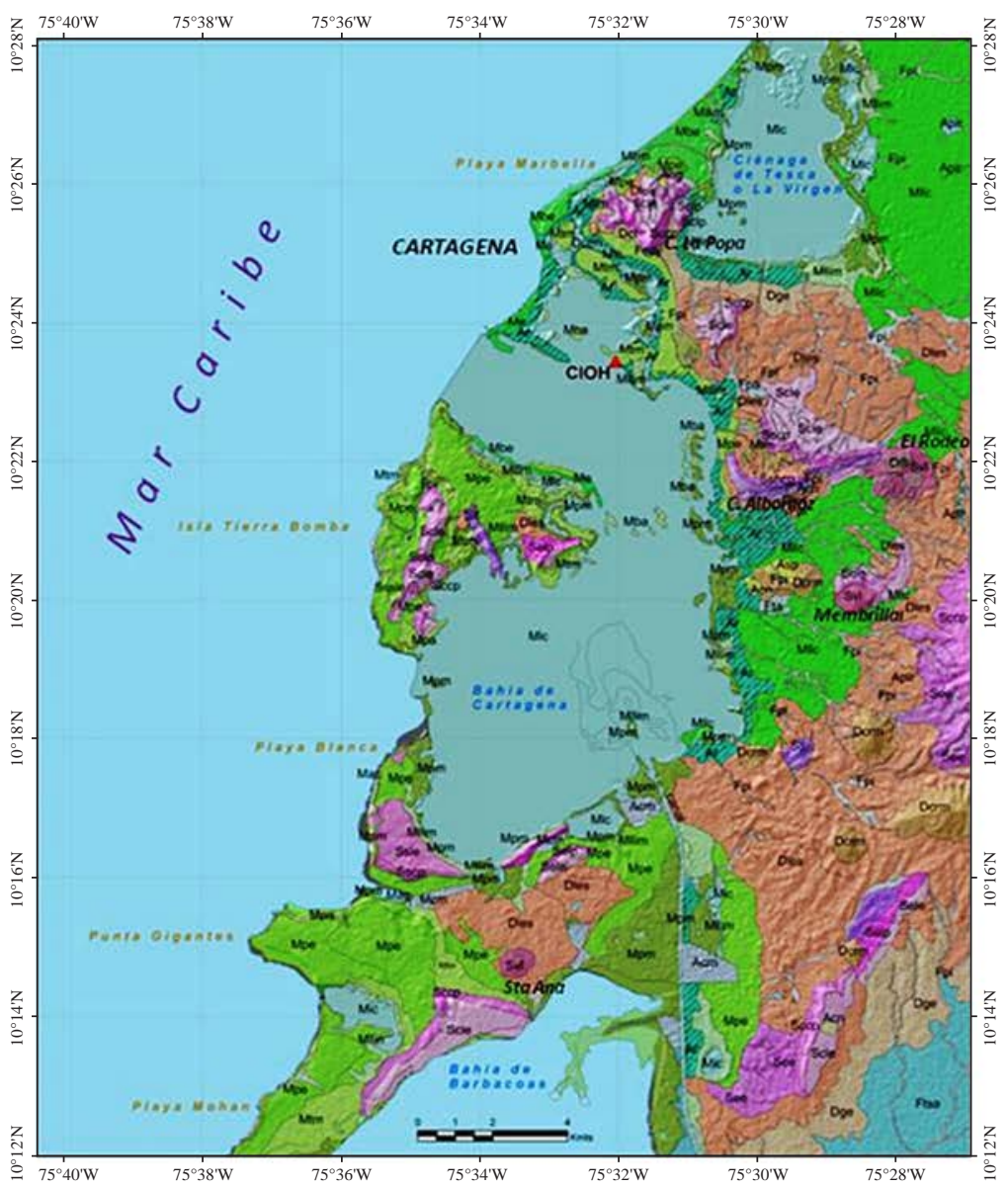

\begin{tabular}{|c|l|}
\multicolumn{2}{|c|}{ Mapa Geomorfológico } \\
\hline \multicolumn{2}{|c|}{ Unidades y subunidades } \\
\hline \multicolumn{2}{|c|}{ Origen antrópico } \\
\hline Acn & Canteras \\
Apir & Represas y Jagueyes \\
\hline Arylh & Rellenos \\
\hline \multicolumn{2}{|c|}{ Origen fluvial } \\
\hline Ftsa & Terrazas fluviales subactuales \\
Fpi & Planos y llanura de inundación fluvial \\
\hline \multicolumn{2}{|c|}{ Origen marino y costero } \\
\hline Mdf & Deltas de flujo mareal \\
Me & Espigas o flechas \\
\hline Mtm & Terrazas marinas \\
Mba & Bajos arrecifales \\
Mlc & Lagunas costeras \\
\hline Mllc & Llanuras costeras \\
\hline Mllim & LLanuras intermareales \\
\hline Mbe & Barras espigas \\
Mpm & Llanuras de manglar \\
Mpe & Plataformas de abrasión elevadas \\
\hline \multicolumn{2}{|c|}{ Origen denudacional } \\
\hline Dcrm & Cerros remanentes \\
Dlfl & Flujos de lodo actuales \\
\hline Dge & Glacis de erosión \\
Dles & Lomas \\
\hline \multicolumn{2}{|c|}{ Origen morfoestructural } \\
\hline Ssan & Sierras anticlinales \\
Sele & Ladera estructural de espinazo \\
\hline Selp & Ladera de contrapendiente de espinazo \\
See & Espolón estructural \\
\hline Scle & Ladera estructural de cuesta \\
\hline Sccp & Ladera de contrapendiente de cuesta \\
Ssle & Ladera estructural de sinclinal \\
Sscp & Ladera de contrapendiente de sinclinal \\
Sshcp & Ladera estructural Sierra Homoclinal \\
\hline Sshle & Ladera contrapendiente Sierra Homoclinal \\
\hline Svl & Volcán de lodo \\
\hline Sme & Escarpe de meseta \\
Slf & Lomo de falla \\
\hline
\end{tabular}

Figura 13. Mapa geomorfológico de los alrededores de la Bahía de Cartagena de Indias. Nótese la pluma de sedimentos provenientes del canal del Dique por el costado SE de la bahía, y la ubicación de la estación GPS del CIOH en la Isla de Manzanillo. Tomado y Modificado de Carvajal, et al. (2011) y Carvajal (2017).

occidente de Cartagena, donde se han encontrado por lo menos cinco sitios con emanaciones de fluidos y gases (Ricaurte, et al., 2004).

Llama la atención, asimismo, la presencia de corales vivos en isla Arena, al occidente de Pueblo Nuevo, Bolívar, en donde las comunidades coralinas crecen a pesar de las condiciones adversas de alta sedimentación asociada con los materiales traídos por el río Magdalena. Se han detectado comunidades coralinas al sur de la Bahía de Cartagena que se conservan en muy buenas condiciones a pesar de los altos aportes de sedimento del Canal del Dique, lo cual es atribuible a posibles emanaciones de lodos y gases en el sector y debe ser motivo de futuras investigaciones en la región.

\section{Conclusiones}

El análisis comparativo de las líneas de costa antiguas y modernas evidenció que en el sur de la Bahía de Cartagena se han perdido islas completas y grandes sectores de manglar costero. Esta situación expone a la línea de costa y a los terrenos de bajamar de Cartagena a una inundación sistemática, gradual y constante, mucho más acelerada que la media global, la cual es el resultado de dos fenómenos conocidos: el aumento del nivel del mar debido al calentamiento global y la subsidencia del terreno. De ahí la necesidad de implementar, en el futuro inmediato, un programa que estabilice la línea de costa, con proyecciones a largo plazo sobre la cota de inundación (Andrade, et al., 2013), y permita la disipación de la energía del oleaje, para así garantizar la supervivencia de la ciudad (Nicolae, et al., 2008). La continuación del proceso iniciado por el Servicio Geológico Colombiano y la DIMAR, orientado a la conexión geodésica de los mareógrafos para establecer los movimientos de orden vertical que se puedan experimentar en la posición de estos instrumentos, es fundamental.

Igualmente importante es contemplar el diapirismo de lodo como una de las causas de los procesos de subsidencia local del terreno en la región de Cartagena. En este sentido, es de vital importancia implementar una red de monitorización mediante técnicas geodésicas tanto terrestres como espaciales para poder observar los movimientos asociados con este tipo de fenómeno. Asimismo, debe iniciarse el estudio de la zona mediante técnicas de geodesia de imágenes basadas en interferometría diferencial con radar de apertura sintética. 
Rev. Acad. Colomb. Cienc. Ex. Fis. Nat. 41(158):94-106, enero-marzo de 2017 doi: http://dx.doi.org/10.18257/raccefyn.360

\section{Conflicto de intereses}

Los autores declaran que el documento es original y no tienen conflicto de intereses de ninguna clase con respecto a su contenido.

\section{Agradecimientos}

Al Servicio Hidrográfico Nacional del Centro de Investigaciones Oceanográficas e Hidrográficas, $\mathrm{CIOH}$, por su ayuda en la georreferenciación de las cartas históricas. A los integrantes del Proyecto GeoRED del Grupo de Investigaciones Geodésicas Espaciales del Servicio Geológico Colombiano, por su apoyo en la instalación, procesamiento y análisis de datos. Al doctor Álvaro Santamaría por la indicación sobre los datos de GPS diferencial del sistema SONEL, y a los revisores de la Revista de la Academia por las críticas constructivas.

\section{Referencias}

Andrade, C.A. \& Franco, R. (2016). Sobre la desaparición de Isla Abanico en Bocachica, Bahía de Cartagena de Indias, Colombia. Paisajes desde proa. Arqueología subacuática de los canales de Manzanillo y Bocachica, Cartagena de Indias. ISBN 978-958-59506-0-3.

Andrade, C.A., Thomas, Y.F., Nicolae-Lerma A., Durand P., Anselme, B. (2013). Coastal flooding hazard related to storm surge events in Cartagena de Indias, Colombia. Journal of Coastal Research. 29 (5): 1126-1136.

Andrade, C.A. (2008). Cambios recientes del nivel del mar en Colombia. En: J.D. Restrepo (editor), Deltas de Colombia: morfodinámica y vulnerabilidad ante el Cambio Global. Fondo Editorial Universidad EAFIT, COLCIENCIAS, pp. 101-121.

Blas de Lezo (1741). Diario de lo acaecido en Cartagena de Indias desde el día 13 de marzo de 1741 hasta el 20 de mayo del mismo año, que remite a S.M. D. Blas de Lezo. En: Arrazola, R., Historial de Cartagena. Cartagena: Editorial Hernández, 1961, pp. 291-321.

Blewitt G. \& Lavallée D. (2002). Effect of annual signals on geodetic velocity, J. Geophys. Res. 107 (B7): ETG 9-1ETG 9-11. doi: 2145, 10.1029/2001jb000570

Bos, M.S., Fernandes, R.M.S., Williams, S.D.P., Bastos, L. (2013). Fast Error Analysis of Continuous GNSS Observations with Missing Data. J. Geod. 87 (4): 351-360. doi: 10.1007/s00190-012-0605-0

Briceño L. \& Vernette G. (1992). Manifestaciones del diapirismo arcilloso en el margen colombiano del Caribe. Revista Geofísica Colombiana. 1: 21-30.

Carvajal, J. H. (2017a). Características del "volcanismo de lodo" del Caribe central colombiano. Informe Servicio Geológico Colombiano, en proceso de publicación. 82 p.

Carvajal J.H. \& Mendivelso D. (2017). Catálogo de "Volcanes de lodo". Caribe Central colombiano. Informe Servicio Geológico Colombiano, en proceso de publicación. Bogotá: INGEOMINAS. 54 p.

Carvajal, J. H. (2016). Mud diapirism in the Central Colombian Caribbean Coastal zone. En: Hermelin, M. (editor). Landscapes and Landforms of Colombia. Springer International Publishing. pp. 35-53.
Carvajal J.H. \& Calderón Y. (2013). La actividad eruptiva del volcán de lodo El Rodeo al sureste del casco urbano de Cartagena de Indias. Informe de atención técnica. Servicio Geológico Colombiano. 60 p.

DIMAR- CIOH (2011). Carta Náutica 264 - Entrada a la Bahía de Cartagena, $3^{a}$ edición.

De Ulloa, A. (1735). Plano de la Bahía de Cartagena de 1735. Copia digital. Madrid: Ministerio de Cultura. Dirección General del Libro, Archivos y Bibliotecas.

Duque-Caro, H. (1979). Major structural elements and evolution of Northwestern Colombia. En: J. S. Watkins, L. Montadert \& P. W. Dickerson (Editores): Geological and Geophysical Investigations of Continental Margins. Am. Assoc. Petr. Geol. Mem. Memoir. 29: 329-351.

Duque H. (1984). Estilo estructural, diapirismo y episodios de acrecimiento del terreno Sinú - San Jacinto en el noroccidente de Colombia. Boletín Geológico INGEOMINAS. 27 (2): 1-29.

Gayet J. \& Vernette G. (1989). Les lagunes côtières. Bull. Inst. Geol. Bassin d'Aquitaine, Bordeaux. 45: 107-121.

Gravelle M., Prouteau E., Wöppelmann G. (2015). Progress Report on the GNSS at Tide Gauge Activities: SONEL Data Holdings \& Tools to access the data. GLOSS-GE Meeting, 21 October - 23 October 2015, Goa, India, 19 p.

He X., Montillet J.-P., Rui Fernandes R., Bos M., Yu K., Hua X., Jiang W. (2917). Review of current GPS methodologies for producing accurate time series and their error sources. Journal of Geodynamics. 106: 12-29. doi: 10.1016/j.jog. 2017.01.004

Herrera y Sotomayor J. \& Hoyo y Abarca C. (1721). Mapa de la Bahía de Cartagena, Biblioteca Nacional de España. Fecha de consulta: febrero de 2017. Disponible en: http://bdh-rd. bne.es/viewer.vm?id=0000031631

Herrera y Sotomayor, J. y J.A. de Ulloa (1735) Plano de la Cyudad y Bahya de Cartagena de las Yndias Situado a los 300 Gs. 41 Ms. de Longytud Prymer Merydo. el Pyco de Teneryfe y 10 Grs. 27 Ms. de Latytud Boreal en el Nuevo Reyno de Granada. febrero de 2017. Disponible en: http://bdh-rd.bne. es/viewer.vm?id=0000020691.

Herring, T., King, R., McClusky, S. (2010a). Gamit and Globk Reference Manuals, Release 10.3, Mass. Inst. of Technol., Cambridge.

Herring, T., King, R., McClusky, S. (2010b). Introduction to Gamit/Globk Reference Manual Global Kalman Filter VLBI and GPS Analysis Program. Release 10.3, Mass. Inst. of Technol., Cambridge,

Intergovernmental Oceanographic Commission of UNESCO. (2006). Manual on Sea-level Measurements and Interpretation, Volume IV: An update to 2006. Paris, Intergovernmental Oceanographic Commission of UNESCO. 78 p.

International Panel for Climate Change (2013). Informe de evaluación del cambio climático. Fecha de consulta: ?. Disponible en: www.ipcc.ch/report/ar5/wg1/

Kellogg J., Toto E., Cerón J. (2005). Structure and tectonics of the Sinú - San Jacinto accretionary prism in Northern Colombia. X Congreso Colombiano de Geología. pp 1-10.

Mantilla A. M., Jentzsch G., Kley J., Alfonso-Pava C. (2009). Configuration of the Caribbean margin: Constraints from 2D seismic reflection data and potential field's interpretation. En: Lallemand, S. y Funicielo, F. Editores. Subduction zones geodynamics. Berlin, Heidelberg: Springer-Verlag, pp. 247-271. 
Martínez I., Yokoyama Y., Gómez A., Delgado A., Matsuzaki H., Rendón E. (2010). Late Holocene marine terraces of the Cartagena Region, southern Caribbean: The product of neotectonism or a former high stand in sea level? J. South American Earth Science. 29 (2): 214-224. doi: 10.1016/j. jsames.2009.08.010

Nicolae, A., Thomas, Y.F., Durand, P., Torres, R.R., Andrade, C.A. (2008). Variabilidad del nivel del mar desde 1950 hasta el 2000 y riesgos asociados a episodios de mar de leva en las penínsulas de Bocagrande y Castillogrande, Cartagena de Indias, Colombia. Boletín Científico CIOH. 26: 71-84.

Pabón, J. D. (2003a). El aumento del nivel del mar en las costas y área insular de Colombia. En: El Mundo marino de Colombia, investigación y desarrollo de territorios olvidados. Red de Estudios del Mundo Marino - REMAR, Universidad Nacional de Colombia, pp.75-82.

Pabón, J.D. (2003b). El cambio climático global y su manifestación en Colombia. Cuadernos de Geografía. XII (1-2): 111-119.

Pabón J.D. \& Lozano J.A. (2005). Aspectos relacionados con las estimaciones globales y regionales del ascenso del nivel del mar y su aplicación a Colombia. Cuadernos de Geografía. 14: $97-106$.

Rangel E.S. \& Montealegre J.E. (2003). Análisis de las series del nivel del mar en el Pacífico colombiano y su relación con el cambio climático. Meteorología Colombiana. 7: 53-66.

Ricaurte C., Domínguez J. G., Mayo G., Andrade C., Ospina H. M. y Gutiérrez A. (2004). Nota sobre algunos rasgos geomorfológicos de los bancos de Salmedina. Boletín CIOH. 22: 64-76.

Santamaría-Gómez A., Gravelle M., Collilieux X., Guichard M., Martín-Míguez B., Tiphaneau P., Wöppelmann G. (2012). Mitigating the effects of vertical land motion in tide gauge records using a state-of-the-art GPS velocity field. Global and Planetary Change: 98-99: 6-17.

Santamaría-Gómez A., Bouin M. N., Collilieux X., Wöppelmann G. (2011). Correlated errors in GPS position time series: Implications for velocity estimates. J. Geophys. Res. 116 (B1). doi:10.1029/2010JB007701

Santamaría-Gómez A. (2010). Estimation des mouvements verticaux de l'écorce terrestre par GPS dans un repère géocentrique, dans le Cadre du Projet TIGA, Thèse de
Doctorat de l'Observatoire de Paris, École Doctorale d'Astronomie et d'Astrophysique d'Île-de-France, 211 pp.

Torres, R. R., \& Tsimplis M. N. (2014). Sea level extremes in the Caribbean Sea, J. Geophys. Res. Oceans. 119: 4714-4731. doi: 10.1002/2014JC009929

Torres, R. R. \& Tsimplis M. N. (2013). Sea-level trends and interannual variability in the Caribbean Sea. J. Geophys. Res. Oceans. 118: 2934-2947. doi:10.1002/jgrc.20229

Torres, R., Gómez, J., Afanador, F. (2006). Variación del nivel medio del mar en el Caribe colombiano. Boletín Científico CIOH. 24: 64-72.

Vernette, G. (1985). La plate-forme continentale caraïbe de la Colombie (du débouché du Magdalena au Golfe de Morrosquillo). Importance du diapirisme argileux sur la morphologie et la sédimentation. Thèse Doct. Etat, Bordeaux, 834, 387 p.

Vernette G., Blanc G., Briceño, L., Carvajal, H., Faugeres, C., Gayet, J., Gonthier, E., Griboulard, R., Molina, A. (1988). Manifestaciones tectónicas en márgenes activas. Comparación entre dos sectores del Caribe - Margen colombiana y Prisma de Barbados. Memorias VI Seminario de Ciencias y Tecnologías del Mar. Pp. 240-252.

Vernette, G. (1989). Examples of diapiric control on shelf topography and sedimentation patterns on the Colombian Caribbean continental shelf. Journal of South American Earth Science. 2 (4): 391-400.

Vernette G., Gayet J., Bobier C., Briceño L., Mauffret A., Molina A. (1990). El frente de deformación sur-Caribe en la región de Cartagena. Posición y relación con la plataforma. Memorias del VII Seminario de Ciencias y Tecnologías del Mar. Comisión Colombiana de Oceanografía. pp 195-209.

Vernette G., Mauffret A., Bobier C., Briceño L., Gayet J. (1992) Mud diapirism, fan sedimentation and strike-slip faulting, Caribbean Colombian Margin. Tectonophysics. 202 (2): 335-349. Doi: 10.1016/0040-1951(92)90118-P

Zumberge, J.F., Heflin, M.B., Jefferson, D.C., Watkins, M.M., Webb, F.H. (1997). Precise point positioning for the efficient and robust analysis of GPS data from large networks. Journal of Geophysical Research. 102 (B3): 5005-5017. doi: 10.1029/96JB03860 